\title{
Thirty Meter Telescope Laser Guide Star Facility Design Update
}

\author{
Melissa Trubey $^{1 *}$, Corinne Boyer ${ }^{1}$, Brent Ellerbroek ${ }^{1}$, Lianqi Wang ${ }^{1}$ \\ Kai Wei ${ }^{2}$, Muwen Fan², Changchun Jiang ${ }^{2}, \mathrm{Min} \mathrm{Li}^{2}$, Daoman Rui ${ }^{2}$ \\ ${ }^{1}$ Thirty Meter Telescope International Observatory, Pasadena, California, USA \\ ${ }^{2}$ Institute of Optics and Electronics, Chengdu, China
}

\begin{abstract}
The Laser Guide Star Facility (LGSF), part of the adaptive optics (AO) system of the Thirty Meter Telescope (TMT), will generate multiple artificial laser guide stars, as required by the TMT Laser Guide Star AO systems. The LGSF will project several possible asterisms on sky, generated at first light by up to six $589 \mathrm{~nm}$ sodium lasers and situated on a platform behind the TMT primary mirror. The beams are then transported using beam transport optics around the elevation rotating structure of the telescope, to a center launch location situated behind the TMT secondary mirror. The asterisms are then generated and projected on sky through a laser launch telescope. The following sections will discuss the LGSF's: overall design (II), key design updates (III), and the next steps for the TMT's LGSF System (IV).
\end{abstract}

Keywords: Laser Guide Star, Adaptive Optics, Thirty Meter Telescope, Beam Transfer Optics

\section{INTRODUCTION}

As discussed in the abstract, this paper provides an overview of the current design and the progress made towards developing the preliminary design of the first light Laser Guide Star Facility (LGSF) ${ }^{[3]}$, which is part of the adaptive optics (AO) system of the Thirty Meter Telescope (TMT) ${ }^{[1]}$. The LGSF is responsible for generating the artificial guide stars required by the first light Adaptive Optics (AO) system and by future AO instruments. The LGSF uses up to 9 sodium lasers to generate and project up to 4 identified LGS asterisms from a laser launch telescope located behind the TMT secondary mirror. Section 2 of this document provides an overview of the LGSF design. Section 3 will cover the key updates made since the last AO4ELT conference. Section 4 will cover the next steps for the LGSF system.

\section{LGSF SYSTEM OVERVIEW}

\subsection{Design Overview}

The Institute of Optics and Electronics (IOE) team in Chengdu (China) is developing the LGSF preliminary design with the support of the TMT AO team. The LGSF System consists primarily of 3 sub-systems:

- The Laser System (LAS), comprised of the sodium lasers with their electronics and cooling system, which generate the LGS beams. The Laser System is mounted to the inside of the $-X_{\mathrm{ECRS}}$ Elevation Journal and on the Laser Platform.

- The Beam Transfer Optics (BTO) Optical Path, comprised of all components necessary for beam transport and conditioning, which transports the laser beams from the outputs of the Laser System to the LGSF Top End. The LGSF Beam Transfer Optics are mounted onto the Telescope Elevation Journal and the Telescope Elevation Truss Members.

- The LGSF Top End (LGSF TE), which is comprised of all the components necessary for the LGS asterism creation and projection to the sky, as well as the LGS acquisition system and the aircraft safety system. The LGSF Top End is located behind the TMT secondary mirror and is attached to the Telescope Elevation Star Structure.

\footnotetext{
* mtrubey@tmt.org; www.tmt.org
} 


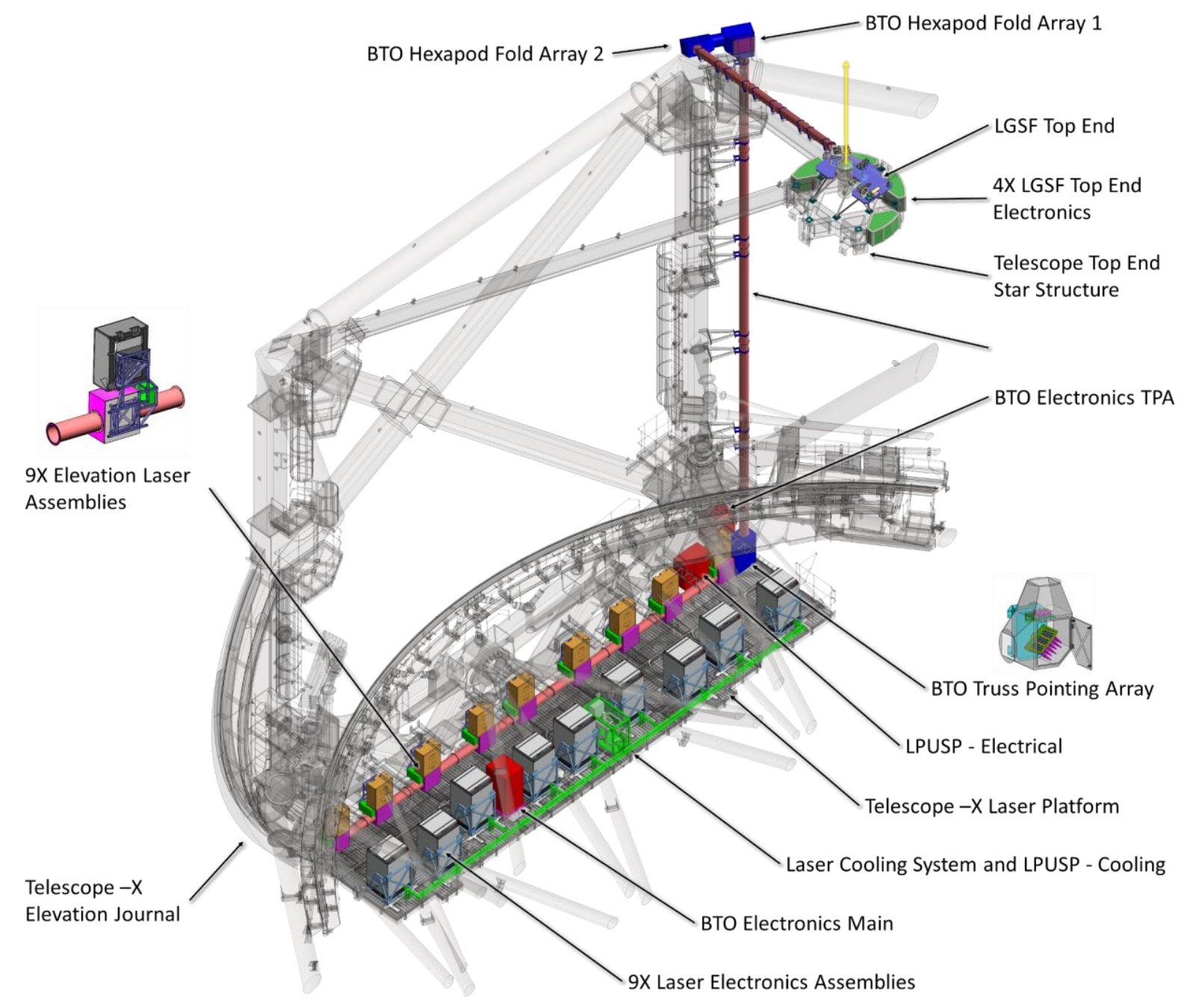

Figure 1: Laser Guide Star Facility Overall Model on the TMT Elevation Structure

\subsection{The LGSF asterisms}

The LGSF will generate the following 4 asterisms on the sky as shown in Figure 2:

- The Multi Conjugate AO (MCAO) asterism, which consists of 6 LGS, 5 LGS equally spaced on a circle of radius of 35 arcsec and one additional on-axis LGS. This asterism will be used by the first light Narrow Field Infrared Adaptive Optics System (NFIRAOS) ${ }^{[2]}$, which will feed up to 3 near IR instruments for spectroscopy and imaging.

- The Laser Tomography AO (LTAO) asterism, which consists of 3 LGS, equally spaced on a circle of radius of 70 arcsec. This asterism is dedicated to instruments for narrow field mid IR spectroscopy and imaging also referred as Mid InfraRed Adaptive Optics System (MIRAO).

- The Multi Object AO (MOAO) asterism, which consists of 8 LGS, 3 LGS equally spaced on a circle of radius of $70 \mathrm{arcsec}$ and 5 LGS equally spaced on a circle of radius of $150 \mathrm{arcsec}$. This asterism is dedicated to moderate field, near IR multi-object IFU spectroscopy.

- The Ground Layer AO (GLAO) asterism, which consists of 4 LGS located at the corners of a 240 arcsec by 360 arcsec rectangle offset by 288 arcsec along the short edge. This asterism is dedicated to wide field optical spectroscopy. 
In addition, several design goals have been defined:

- As a goal, the LGSF will be designed to generate the MCAO asterism with any 6 of the available lasers.

- As a goal, the LGSF will be designed to generate the LTAO asterism with any 3 of the available lasers.

- As a goal, the LGSF will be designed to generate the GLAO asterism with any 4 of the available lasers.

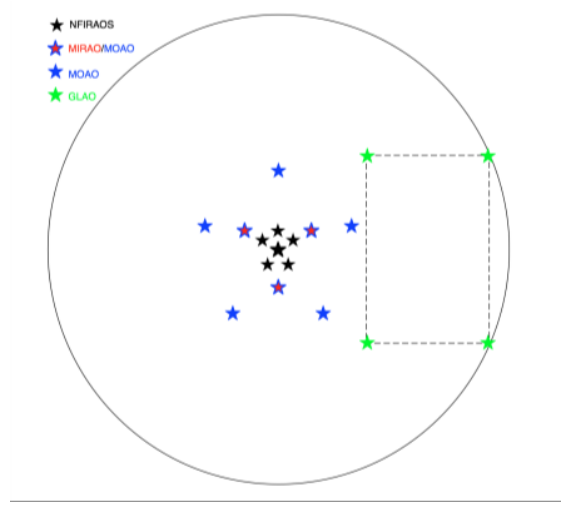

Figure 2: LGSF asterisms

\subsection{LGSF Optical Layout}

The optical layout of the LGSF is given in Figure 3.

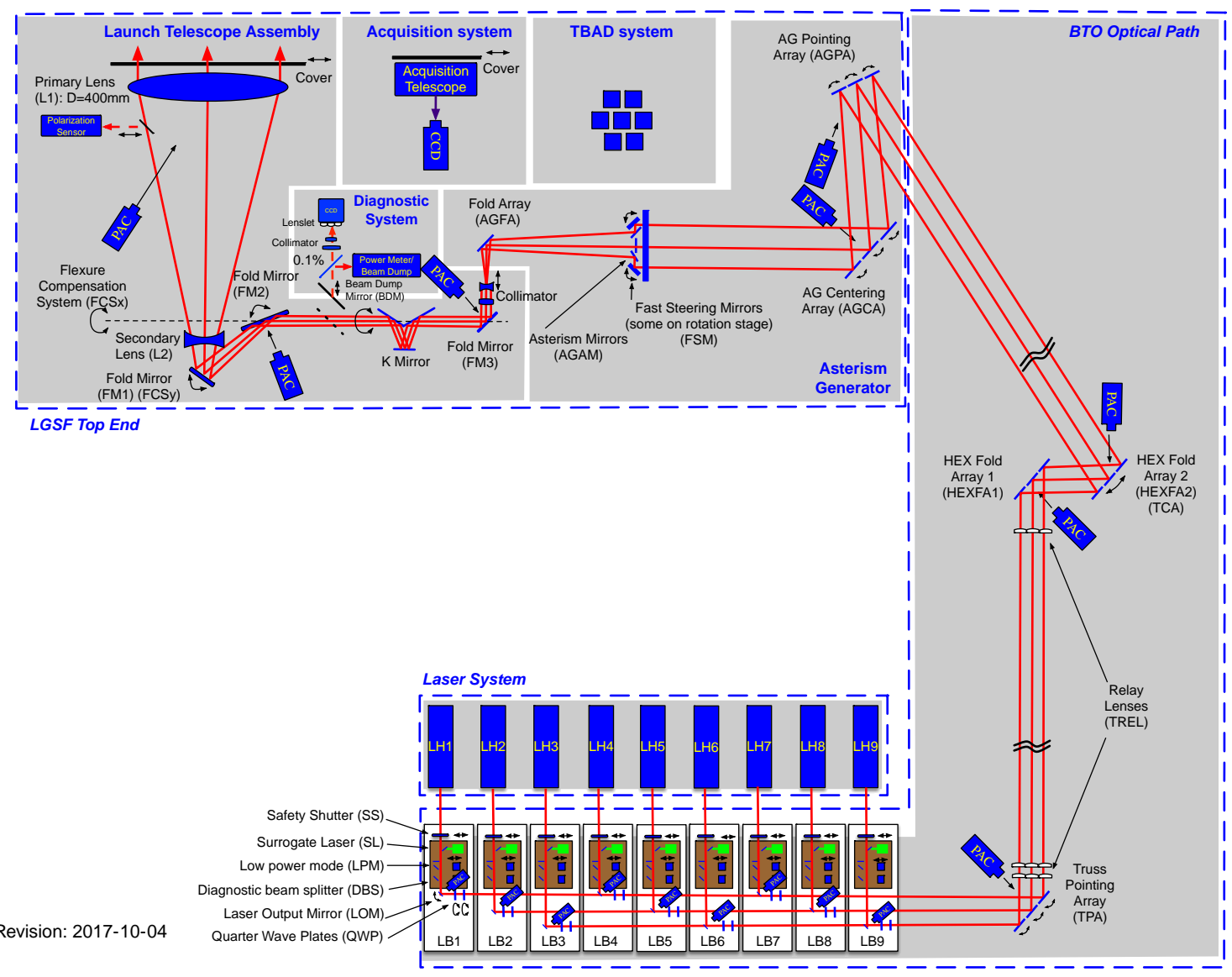

Figure 3: LGSF Optical Layout 
At first light, only six lasers will be installed to generate the MCAO or NFIRAOS asterism. Lasers 1, 5 and 9 (L1, L5 and L9 as described in Figure 3) will not be installed. As a result, if one of the six lasers is not available, the LGSF is designed to redirect one or more laser beams so a reduced MCAO asterism without a center LGS beam can be projected on the sky.

\subsection{Laser Bench (LB)}

Each laser beam generated by a $20 \mathrm{~W}$ sodium laser (referenced LH1 to LH9 in Figure 3) is propagated through a laser bench (referenced LB1 to LB9 in Figure 3) which includes the following optical components:

- An entrance window

- A safety shutter and associated beam dump

- A low power mode mechanism consisting of a thin film polarizer and a half wave plate

- A diagnostic beam splitter and beam splitter camera

- A surrogate sodium laser and surrogate fold mirror

- A laser output mirror to fold the vertical beam horizontally

- A pre-alignment camera

- Two quarter wave plates to ensure circular polarization of the laser beam on the sky

The laser bench can be configured in one of the following three positions:

- High power mode: this is the nominal configuration for observation. In this case, the stage supporting the low power mode mechanism, the diagnostic beam splitter and the surrogate fold mirror is retracted. The $20 \mathrm{~W}$ laser beam is propagated directly to the laser output mirror and then folded horizontally though the two quarter wave plates.

- Low power mode: in this mode, the stage supporting the low power mode mechanism, the diagnostic beam splitter and the surrogate fold mirror is inserted so the $20 \mathrm{~W}$ laser beam goes through the low power mode mechanism and then through the diagnostic beam splitter. A small fraction of the beam is sent to the diagnostic camera, and the remainder of the beam is then sent to the laser output mirror similarly to the high-power mode.

- Surrogate mode: in this mode, the stage supporting the low power mode mechanism, the diagnostic beam splitter and the surrogate fold mirror is inserted so the surrogate fold mirror is in path and can fold the surrogate laser beam toward the laser output mirror. In this case, the $20 \mathrm{~W}$ laser is not propagating or is shuttered.

To format the 9 laser beams into a compact 3x3 laser beam array of 140mm on the side, the laser output mirrors can take 3 vertical positions while the benches are spaced at 3 different offset positions from the elevation journal by grouping of 3 lasers. Figure 4 illustrates the three laser bench configurations with the three laser output mirror positions.

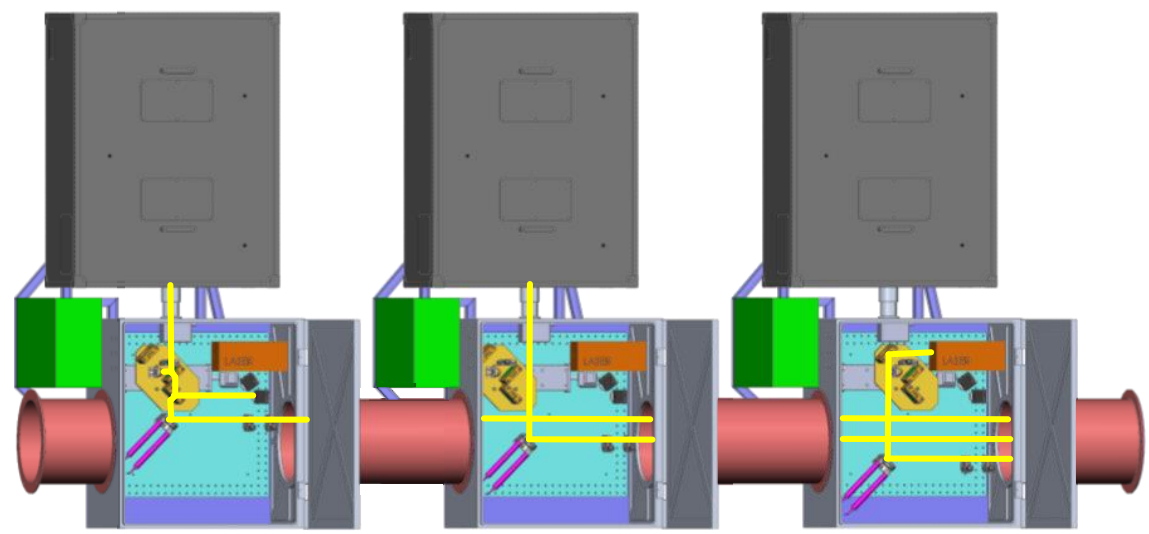

Figure 4: Laser bench configuration (Left: low power mode, middle: high power mode, right: surrogate mode) 
Finally, the laser output mirrors are actuated for tip and tilt control in order to compensate for telescope flexure when the telescope is moving in elevation. The quarter wave plates will be controlled individually via a Lookup Table (LUT) based on the telescope elevation orientation.

\subsection{The Truss Pointing Array (TPA)}

The $3 \times 3$ laser beam array is folded vertically at the output of the laser platform with the Truss Pointing Array (TPA). The TPA consists of an array of $3 \times 3$ individual mirrors, each of them actuated for tip and tilt control in order to compensate for telescope flexure when the telescope is moving in elevation.

\subsection{The Relay Lens System}

A set of three relay lenses in each laser beam expands each laser beam diameter from $3 \mathrm{~mm}$ to $5 \mathrm{~mm}$ and controls the diffraction spread of the Gaussian laser beams over the 50+ $\mathrm{m}$ trip to the telescope top end in order to reimage the laser beams from the laser output ports onto the entrance pupil of the Laser Launch Telescope.

\subsection{Hexapod Fold Arrays;}

A set of two arrays of $3 \times 3$ individual mirrors, called Hexapod Fold Array 1 (HEXFA1) and Hexapod Fold Array 2 (HEXFA2), allow to relay the $3 \times 3$ compact laser beams along the hexapod leg of the telescope toward the top end. The second array, HEXFA2, is globally actuated for tip and tilt control in order to compensate for telescope flexure when the telescope is moving in elevation.

\subsection{The Asterism Generator}

The Asterism Generator (AG) contains the following optical components:

- The asterism generator pointing array (AGPA)

- The asterism generator centering array (AGCA)

- The asterism generator bracket itself supporting:

○ The fast Steering Mirrors (FSM), some mounted on rotational stages

- The asterism generator asterism mirrors

- The asterism generator fold mirror (AGFM)

The AGPA, an array of $3 \times 3$ individual mirrors, allows to fold the beams toward the AGCA. The individual mirrors are actuated for tip and tilt control in order to point the laser beams onto the FSMs of the AG.

The AGCA, consists of an array of $3 \times 3$ individual mirrors. The individual mirrors are actuated for tip/tilt control in order to center (in tandem with AGPA) the laser beams onto the FSMs of the AG.

The primary function of the $\mathrm{AG}$ is to create the 4 required asterisms by manipulating the $3 \times 3$ compact laser beam array into the required asterisms using the FSMs, their rotational stages, and the asterism generator asterisms mirrors (AGAM). The current design of the AG is limited as follows:

- The AG can only generate the 4 identified asterisms described in section 2.2, i.e. MCAO, LTAO, MOAO, GLAO. Therefore only 8 beams are required. It is not possible with the current design to generate an undefined asterism using 9 beams.

- The AG generates the GLAO asterism as a centered asterism and the Laser Launch Telescope offsets it before launching it on the sky. The reason to generate the GLAO asterism in two steps is to reduce the size of the optics between the AG and the laser Launch Telescope Assembly. 


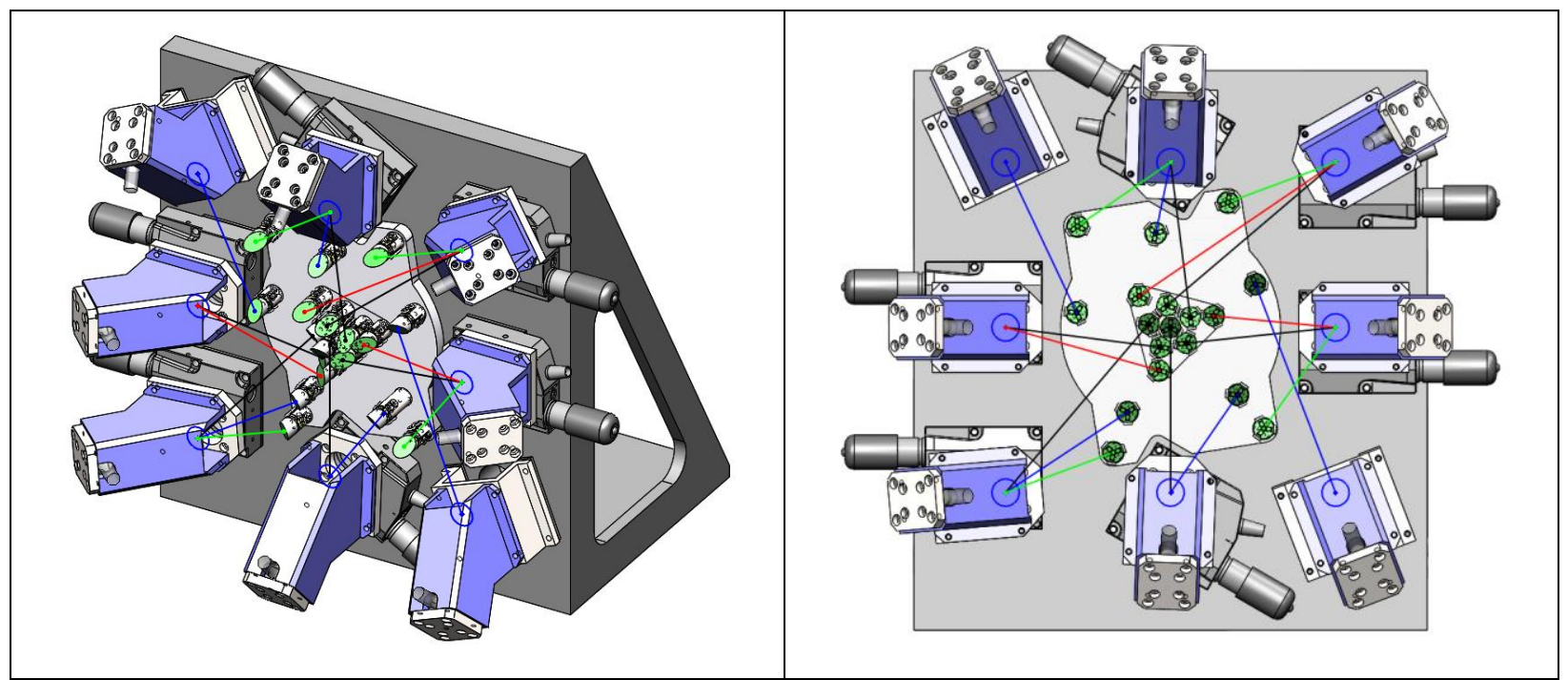

Figure 5: Asterism Generator Layout.

As illustrated in Figure 5, only six of the FSMs are mounted on rotational stages, and these rotational stages can take up to 3 positions in order to generate the 4 asterisms. The other two FSMs are mounted on a fixed mount. The FSMs themselves are fast steering mirrors which are used to compensate Laser Guide Star (LGS) high speed bandwidth pointing jitter as measured by the associated LGS wavefront sensor in the corresponding AO system.

The Asterism Generator Fold Mirror (AGFM) consists of a 75mm diameter mirror which folds the laser beams into the Laser Launch Telescope Assembly.

\subsection{The Laser Launch Telescope Assembly}

The Laser Launch Telescope Assembly (LTA) is based on a refractive design (see Figure 6) and contains the following optical components:

- The collimator

- $\quad$ The LTA fold mirror 3 (FM3)

- The K-Mirror

- The laser launch telescope optical tube, mounted on a pivot mount and including the following components:

- The LTA fold mirror 2 (FM2)

- The LTA fold mirror 1 (FM1)

- The Laser Launch telescope composed of the two lenses (LLT L1 and LLT L2)

The collimator is composed of two lenses and acts as a first magnification module with a magnification factor of 6 .

FM3 is a $110 \mathrm{~mm}$ diameter fold mirror and is used to fold the collimated beams onto the K-Mirror.

The K-Mirror acts as an image rotator and maintains a fixed asterism in the LGS wavefront sensor focal plane. It rotates as one unit and as a function of the telescope elevation angle and azimuthal angle for some asterisms.

The Laser Launch Telescope is composed of two fold mirrors (FM2 and FM1) and two lenses, which acts as the second magnification module with a magnification factor of 8 . The diameter of the LLT is $400 \mathrm{~mm}$ and allows to expand the $30 \mathrm{~mm}$ Gaussian beams into a $1 / \mathrm{e} 2$ diameter of $240 \mathrm{~mm}$.

The entire Laser Launch Telescope is mounted on a pivot mount (Referred as the Flexure Compensation System - FCS) to compensate for large telescope flexure around the X axis only. FM1 is actuated for tip/tilt control and allows to compensate for telescope flexure around the $\mathrm{Y}$ axis and also to compensate for medium bandwidth pointing jitter as measured by the associated LGS wavefront sensor in the corresponding AO system. 
In addition, FM1 is used to offset the GLAO asterism. Finally, to compensate for pupil shift introduced by FM1, FM2 is actuated for tip/tilt control.

There may be a need to adjust the focus of the LTA to compensate for the change of the sodium layer with the telescope elevation. One possible solution would be to mount the LLT L2 onto a small piston stage.

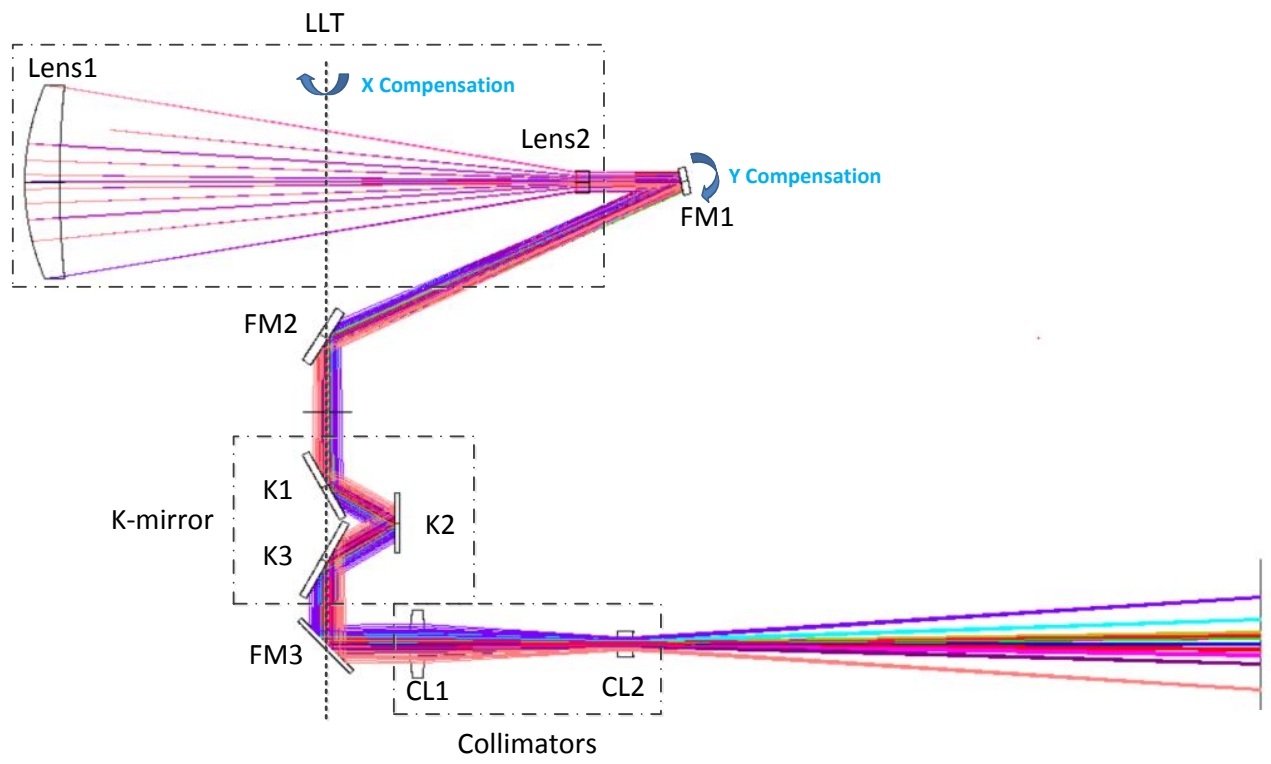

Figure 6: Laser Launch Telescope Assembly Optical Design.

\subsection{The Diagnostic System}

The Diagnostic System (DS) includes the following optical elements:

- $\quad$ The Beam Dump Mirror (BDM)

- The beam splitter

- The power meter and beam dump

- The DS wavefront sensor (WFS) and associated optics including:

○ The DS collimator

$\circ$ The lenslet array

- The camera including the CCD.

The Beam Dump Mirror consists of a large mirror which, once inserted into the path, directs the laser beams into a beam splitter. These beams are then sent through a power meter and a small fraction of the beams are transmitted to the DS WFS.

The purpose of the DS WFS is to measure the pointing and centering errors of all laser beams, as well as beam quality. These errors are corrected using the FSM, AGPA and AGCA mirrors in real time.

\subsection{The LGS Acquisition System}

The purpose of the Laser Guide Star Acquisition system is to verify the position and geometry of the projected asterism on the sky. The Acquisition System consists mainly of a 100mm telescope and a CCD.

\subsection{The Pre-Alignment Cameras}

The Pre-Alignment Cameras are located at different locations of the LGSF optical path as described in Figure 3 and are used to do a rough alignment of the LGSF Optical Path. 


\section{DESIGN UPDATES}

\subsection{Optical Layout Updates}

Several major changes to the optical design have been implemented as follows:

- The LLT design was changed from an off axis reflective design to refractive design. The change from a reflective to a refractive Laser Launch Telescope (LLT) was primarily necessitated by the exceedingly tight optical and mechanical tolerances that were found to be necessary for such a design after an optical tolerance study was completed.

- The Top End overall layout was simplified:

- Diagnostic alignment cameras were replaced with diagnostic wave front sensors and pre-alignment cameras,

- Complexity was reduced by removing redundancy of some of the positioning actuators within the AG,

$\circ$ Optics between the asterism generator and the laser launch telescope were reduced by offsetting the large asterism (GLAO) with the laser launch telescope fold mirror 1,

- The previous radial design of the AG was abandoned and replace by a bench design to improve access to its components,

$\circ$ Finally the entire layout of the LGSF top end was modified to provide access to the back of the telescope secondary mirror.

The updated overall mechanical layout for the top end can be seen in Figure 7.

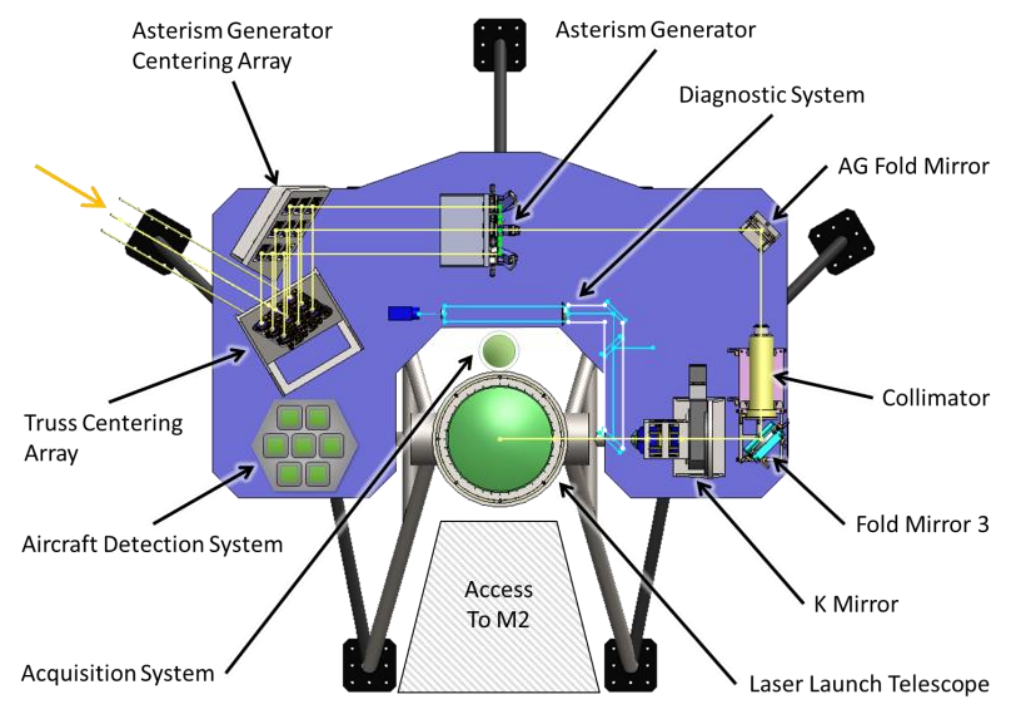

Figure 7. LGSF Top End Mechanical Layout

\subsection{Structure Interface Updates}

Much progress has been made in solidifying the LGSF interfaces with the Telescope Structure, including updating of the space envelopes, attachment interfaces, cabling and piping schematics, services definitions, updated mass budgets, and structural analysis.

\subsection{Local Laser Cooling System}

The laser cooling system design for the LGSF has been updated to provide for an on-platform local solution. The previous cooling system design routed pipes from summit services through the azimuth and elevation cable wraps to the laser system on laser platform. These long lengths of piping, even with insulation, could not deliver chilled water to the 
lasers within the tight temperature tolerances required by the lasers, and also generated heat dissipation under primary mirror beyond the allowable budget.

The new on-platform cooling system, situated between lasers electronics units 5 and 6 , allows accurate control of the cooling temperature though utilization of short insulated piping lengths, and reduces thermal dissipation under the primary mirror by being fed with summit supplied variable temperature chilled water (VTCW), and returning the VTCW after passing across a heat exchanger prior to return.

The Laser Cooling System has two modes of operation, as shown in Figure 8:

- Daytime warm-up local bypass circuit

- Passive observing operation delivered by to summit services

During the daytime warm-up mode, the local circuit is created by closing off the supply and return from summit services and opening the local circuit bypass. The chilled water contained in the piping is then circulated by the on-platform pump, across the heat exchanger, though the heater, distributed to all laser electronics units desired, and then returned back across the heat exchanger and though the pump. This allows for expeditious heating and temperature stabilization of the laser system.

During the passive observing operation mode, the system which is already at temperature, is fed by the summit services supply and return. The local bypass valve is closed and pump is off. The summit supplied chilled water is passed across the heat exchanger, though the heater, distributed to all laser electronics units desired, and then returned back across the heat exchanger and back to summit services. Temperature is maintained though use of the heater and the heat exchanger bypass to ensure VTCW temperature with stability required for lasers.

Of note, the immersion heater is sized so as the laser cooling system could, from a cold start, be fully operational without use of the warm-up mode. However, the warm-up mode was implemented to allow for a more rapid system readiness timeframe.

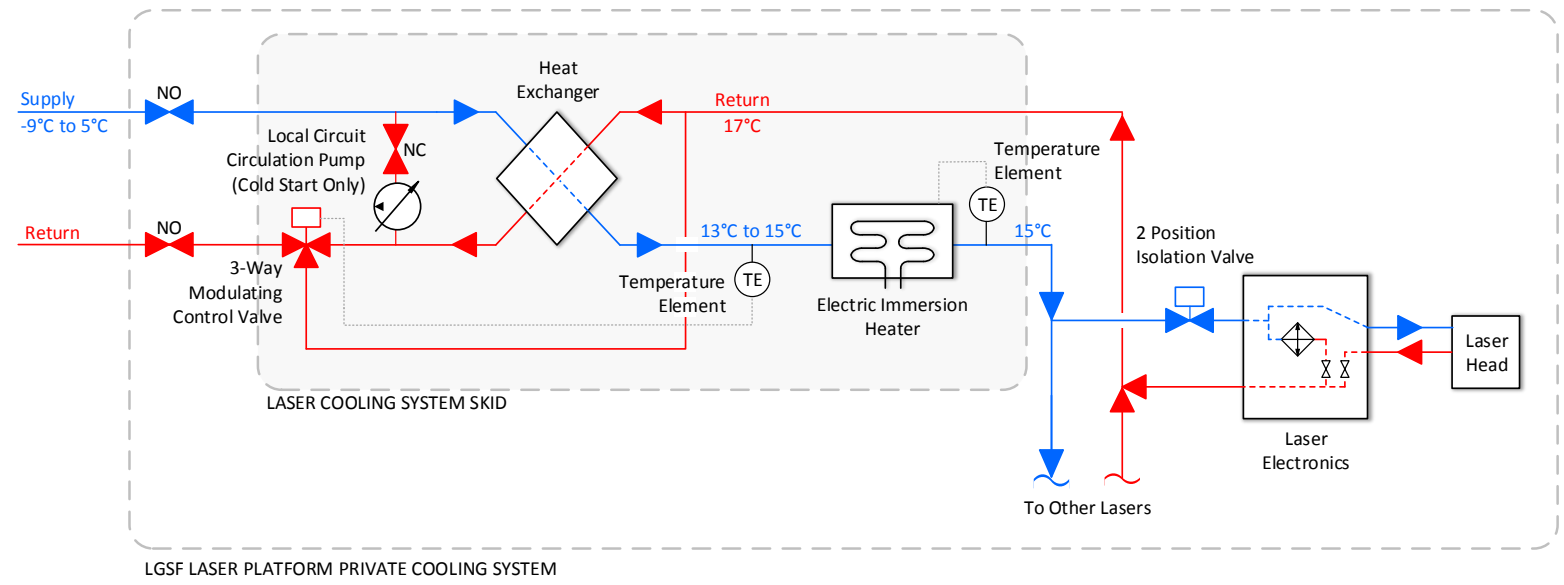

Figure 8: Laser Cooling System Schematic

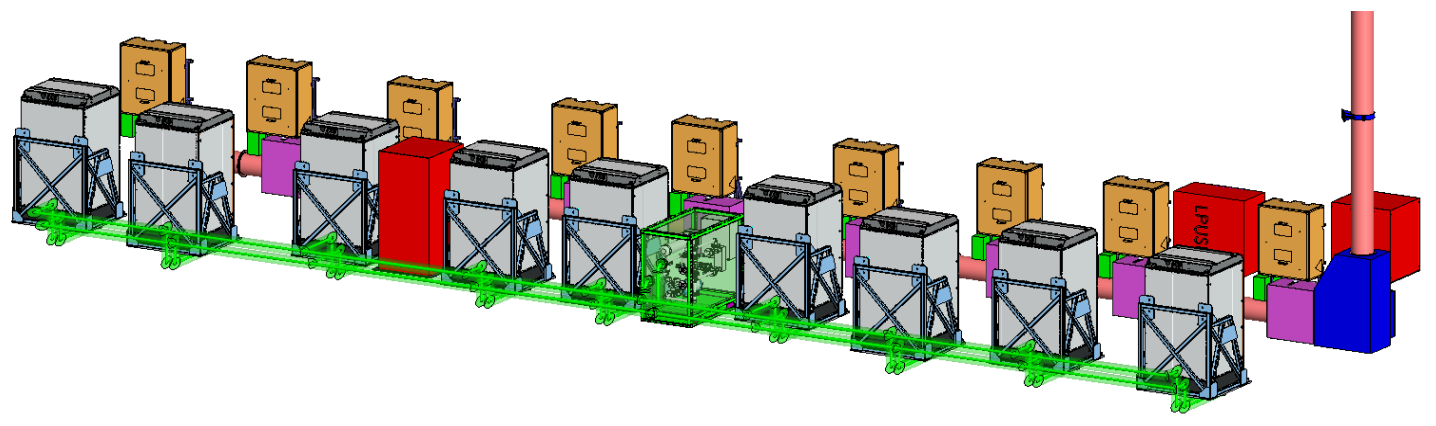

Figure 9: Laser Cooling System situation on Laser Platform 


\section{NEXT STEPS}

The LGSF team is currently preparing for a Preliminary Design Review by March of 2018, primarily for its mechanical, optical, and electronics design. Work currently being done in mechanical design includes: CAD modeling, FEA, component selections, and performance budgets. Current tasks for optical design include finalizing of the design and performance budgets including: tolerances, beam quality, and throughput. At present the electronics design is near preliminary design level, however, some design updates will need to occur for the electronics cooling system due to the change to refrigerant from chilled water. Additional tasks regarding systems engineering will also be addressed.

\section{ACKNOWLEDGMENTS}

The TMT Project gratefully acknowledges the support of the TMT collaborating institutions. They are the Association of Canadian Universities for Research in Astronomy (ACURA), the California Institute of Technology, the University of California, the National Astronomical Observatory of Japan, the National Astronomical Observatories of China and their consortium partners, and the Department of Science and Technology of India and their supported institutes. This work was supported as well by the Gordon and Betty Moore Foundation, the Canada Foundation for Innovation, the Ontario Ministry of Research and Innovation, the National Research Council of Canada, the Natural Sciences and Engineering Research Council of Canada, the British Columbia Knowledge Development Fund, the Association of Universities for Research in Astronomy (AURA), the U.S. National Science Foundation, and the National Institutes of Natural Sciences of Japan.

\section{REFERENCES}

[1] Boyer C., "Adaptive Optics Program Update at TMT," AO4ELT5 Proceedings, (2017).

[2] Herriot G., et al., "NFIRAOS," AO4ELT5 Proceedings, (2017).

[3] Boyer C. et al., "The TMT Laser Guide Star Facility," AO4ELT1 Proceedings, (2009). 